\title{
Electromagnetic form factors of the nucleon: New fit and analysis of uncertainties
}

\author{
W. M. Alberico, ${ }^{1}$ S. M. Bilenky, ${ }^{2,3}$ C. Giunti, ${ }^{1}$ and K. M. Graczyk ${ }^{1,4, *}$ \\ ${ }^{1}$ Dipartimento di Fisica Teorica, Università di Torino and INFN, Sezione di Torino, I-10125 Torino, Italy \\ ${ }^{2}$ BLTP, JINR, RU-141980 Dubna, Russia \\ ${ }^{3}$ Physics-Department E15, Technische Universität München, D-85748 Garching, Germany \\ ${ }^{4}$ Institute of Theoretical Physics, University of Wroctaw, pl. M. Borna 9, PL-50204 Wroctaw, Poland \\ (Received 18 December 2008; revised manuscript received 2 April 2009; published 23 June 2009)
}

\begin{abstract}
Electromagnetic form factors of proton and neutron, obtained from a new fit of data, are presented. The proton form factors are obtained from a simultaneous fit to the ratio $\mu_{p} G_{E p} / G_{M p}$ determined from polarization transfer measurements and to $e p$ elastic cross section data. Phenomenological two-photon exchange corrections are taken into account. The present fit for protons was performed in the kinematical region $Q^{2} \in(0,6) \mathrm{GeV}^{2}$. For both protons and neutrons we use the latest available data. For all form factors, the uncertainties and correlations of form factor parameters are investigated with the $\chi^{2}$ method.
\end{abstract}

DOI: 10.1103/PhysRevC.79.065204

PACS number(s): $13.40 . \mathrm{Gp}, 14.20 . \mathrm{Dh}, 13.60 . \mathrm{Fz}$

\section{INTRODUCTION}

The nucleon electromagnetic form factors are fundamental quantities of great theoretical and experimental importance. The issue of their determination has been revisited in recent years, thanks to the results of several experiments at Bates, Mainz Microtron (MAMI), and the Thomas Jefferson National Accelerator Facility (JLab), which put under question previous analyses based on less precise data and urged the necessity for a new parametrization and a new analysis of the form factors themselves (for a review, see, for example, Refs. [1-3]).

A precise knowledge of the electromagnetic form factors of the nucleon is important for the determination of the axial nucleon form factor in charged current (CC) quasielastic neutrino-nucleon scattering [4] and strange form factors of the nucleon in neutral current (NC) elastic neutrino-nucleon scattering. For example, NC vector form factors that characterize elastic NC scattering are given by the following expressions [5]:

$$
\begin{aligned}
& G_{E}^{\mathrm{NC} ; p(n)}= \pm \frac{1}{2}\left\{G_{E p}-G_{E n}\right\}-2 \sin ^{2} \theta_{W} G_{E p(n)}-\frac{1}{2} G_{E s}, \\
& G_{M}^{\mathrm{NC} ; p(n)}= \pm \frac{1}{2}\left\{G_{M p}-G_{M n}\right\}-2 \sin ^{2} \theta_{W} G_{M p(n)}-\frac{1}{2} G_{M s} .
\end{aligned}
$$

In the above, the dominant terms are the electric $\left(G_{E}\right)$ and magnetic $\left(G_{M}\right)$ form factors of the nucleon. Their precise knowledge is essential to determining the small strange form factors of the nucleon, $G_{E s}$ and $G_{M s}$. Hence, it is obvious that not only a good knowledge of the electromagnetic form factors is required, but also the present level of their uncertainty.

In this paper, we performed new fits of the nucleon electromagnetic form factors. The proton ones are extracted from (i) elastic ep cross section data and (ii) polarization data, providing the $\mu_{p} G_{E} / G_{M}$ ratio ( $\mu_{p}$ being the magnetic moment of the proton). The neutron form factors are extracted from electron-nucleus (typically deuterium and ${ }^{3} \mathrm{He}$ ) scattering processes. The latest experimental data are used.

The proton form factors determined from the measurements of polarization transfer in elastic electron-proton scattering

\footnotetext{
*graczyk@to.infn.it
}

(first appearing between 1999 and 2002) were in significant disagreement with those obtained from elastic ep scattering data via the customary Rosenbluth separation. The main suggestion for solving this inconsistency was to account for two photon exchange (TPE) diagrams [6-9], which should affect the cross section to a greater extent than the polarization data.

This disagreement became even more evident after the new JLab data on $e p$ scattering cross sections [10] appeared. Hence, as already pointed out by several authors (see, e.g., Ref. [11]), a reliable global fit must include the TPE correction; we will explicitly show the effect of TPE on the goodness of the fit (GoF). For a recent review devoted to TPE correction, see Ref. [12].

Several parametrizations of the nucleon form factors have been considered in the literature [11,13-22]. Among these, the older ones have a purely empirical $Q^{2}$ dependence [13-16]:

$G_{E p}\left(Q^{2}\right), \quad G_{M p}\left(Q^{2}\right) / \mu_{p} \sim \frac{1}{1+\sum_{i=1}^{N} c_{i} Q^{i}}, \quad Q=\sqrt{Q^{2}}$.

The specific form of the parametrization may depend on the $Q^{2}$ region. For instance, in Ref. [19] the low- $Q^{2}$ data were analyzed with form factors given by a continued fraction parametrization:

$$
G_{E p}\left(Q^{2}\right), \quad G_{M p}\left(Q^{2}\right) / \mu_{p} \sim \frac{1}{1+\frac{b_{1} Q^{2}}{1+\frac{b_{2} Q^{2}}{1+\cdots}}} .
$$

The newest empirical form factors are constrained to have a proper physical behavior at low- $Q^{2}$ as well as at high- $Q^{2}$. One example is Kelly's parametrization [18], which will be employed in our analysis (see the next section). The form factors depend on powers of the invariant $Q^{2}$, and for large $Q^{2}$ the form factors behave like $1 / Q^{4}$. In Ref. [20], Kelly's parametrization is additionally constrained to satisfy a duality hypothesis, and the low- $Q^{2}$ behavior is described as in Ref. [19].

The electric neutron form factor is usually separately treated and described with a smaller number of parameters [23] (see also Ref. [24]). 
It is also worth mentioning those parametrizations obtained on the basis of the vector meson dominance model. In particular, the parametrization proposed by Lomon [22] seems especially suited to successfully describing the neutron form factor data.

In the present paper, we aim to provide reliable fits of both proton and neutron electromagnetic form factors, by employing a relatively small number of parameters; moreover, one of the major merits of this work is the analysis of errors on the parameters of the fit, which allows one to estimate the present uncertainty on our knowledge of the electromagnetic form factors.

The paper is organized as follows: in Sec. II we consider the proton form factors, by analyzing both the polarization data (Sec. II A) and the cross section data (Sec. II B). Section III is devoted to the neutron form factors, separately considering the electric and the magnetic form factors. Finally, Sec. IV presents a discussion of our results in comparison with previous analyses and the conclusions.

\section{PROTON FORM FACTORS}

In Sec. II A we consider the recent polarization transfer and asymmetry measurement data, which give information on the ratio of the electric and magnetic proton form factors. Then, in Sec. II B, we present the results of the combined fit of the polarization and cross section data.

\section{A. Fit of polarization data}

In this section we consider the direct determination of the ratio of the electric and magnetic proton form factors

$$
\mathcal{R}\left(Q^{2}\right) \equiv \mu_{p} \frac{G_{E p}\left(Q^{2}\right)}{G_{M p}\left(Q^{2}\right)},
$$

which has been obtained with the measurement of the polarization of the recoil proton and with asymmetry measurements. Here $Q^{2} \equiv-q^{2}, q$ being the four-momentum transfer. In the one-photon approximation, $q$ is the four-momentum of the virtual photon.

The recoil polarization technique (see Ref. [3]) has been employed in several $e p$ experiments for a direct measurement of the ratio $\mathcal{R}\left(Q^{2}\right)$. In the laboratory frame, it is given by

$$
\mathcal{R}\left(Q^{2}\right)=-\mu_{p} \frac{P_{t}}{P_{l}} \frac{E+E^{\prime}}{2 M} \tan \left(\frac{\theta}{2}\right),
$$

where $P_{l}$ and $P_{t}$ are the longitudinal and transverse components of the polarization of the recoil proton, $M$ is the proton mass, $E$ and $E^{\prime}$ are the initial and final electron energies, and $\theta$ is the electron scattering angle. The latter is related to $Q^{2}$ according to

$$
Q^{2}=4 E E^{\prime} \sin ^{2}\left(\frac{\theta}{2}\right) .
$$

The ratio $\mathcal{R}\left(Q^{2}\right)$ has been also determined from the measurement of the asymmetry in elastic $e p$ scattering with both polarized beam and target: we have (see Ref. [3])

$$
\frac{\sigma_{+}-\sigma_{-}}{\sigma_{+}+\sigma_{-}}=-2 \mu_{p} \sqrt{\tau(1+\tau)} \tan \left(\frac{\theta}{2}\right) \frac{\mathcal{R} \sin \theta^{*} \cos \phi^{*}+\mu_{p} \sqrt{\tau\left[1+(1+\tau) \tan ^{2}\left(\frac{\theta}{2}\right)\right]} \cos \theta^{*}}{\mathcal{R}^{2}+\mu_{p} \tau / \epsilon},
$$

where $\sigma_{+}$and $\sigma_{-}$are the cross sections for positive and negative electron helicities, respectively, $\theta^{*}$ and $\phi^{*}$ are the polar and azimuthal angles of the target polarization relative to the threemomentum transfer vector $\vec{q}$ and the scattering plane (in the laboratory frame)

$$
\tau \equiv \frac{Q^{2}}{4 M^{2}},
$$

and

$$
\epsilon \equiv\left[1+2(1+\tau) \tan ^{2}\left(\frac{\theta}{2}\right)\right]^{-1}
$$

is the virtual photon polarization.

We consider the recoil polarization and asymmetry data published in Refs. [25-36]. These data are plotted in Fig. 1, together with their error bars, which include the statistical and systematic uncertainties added in quadrature. The $Q^{2}$ range of the data goes from 0.15 to $5.6 \mathrm{GeV}^{2}$. As one can see from
Fig. 1, all data are well described by a linear function in $Q^{2}$ :

$$
\mathcal{R}\left(Q^{2}\right)=c_{0}+c_{1} Q^{2} .
$$

We fitted the data points with this linear function, by minimizing the least-squares function

$$
\chi_{\mathrm{rat}}^{2}=\sum_{j=1}^{N_{\text {rat }}} \frac{\left(\mathcal{R}\left(Q_{j}^{2}\right)-\mathcal{R}_{j}^{\exp }\right)^{2}}{\left(\Delta \mathcal{R}_{j}^{\exp }\right)^{2}}
$$

where $N_{\text {rat }}=65$ is the total number of recoil polarization and asymmetry data points and $\mathcal{R}_{j}^{\exp }$ is the value of the ratio at the squared-momentum transfer $Q_{j}^{2}$, with corresponding uncertainty $\Delta \mathcal{R}_{j}^{\exp }$.

We found the following best-fit values of the parameters:

$$
c_{0}=1.022 \pm 0.005, \quad c_{1}=-0.130 \pm 0.005,
$$




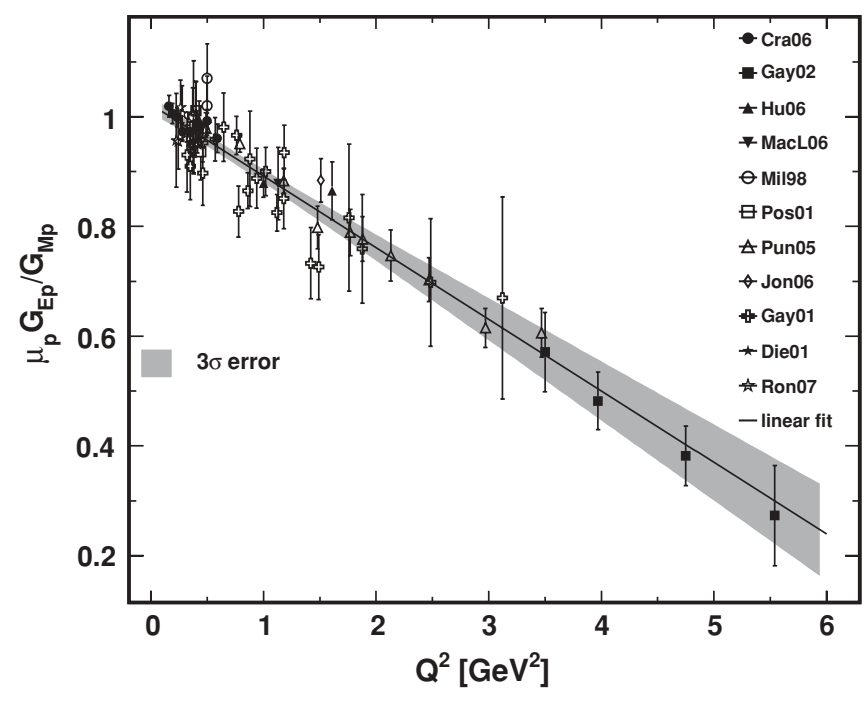

FIG. 1. Linear fit (9) of the recoil polarization and asymmetry measurements of the ratio $\mu_{p} G_{E p} / G_{M p}$. The shadowed area denotes the $3 \sigma$ C.L. region of the fit.

with $1 \sigma$ uncertainties computed from the covariance matrix ${ }^{1}$ (they are given by the square roots of the diagonal elements of the covariance matrix).

The corresponding minimum $\chi^{2}$ is

$$
\left(\chi_{\text {rat }}^{2}\right)_{\min } / \mathrm{NDF}=58.89 / 63,
$$

where NDF $=N_{\text {rat }}$ is the number of degrees of freedom; the goodness of the fit (see Ref. [38]) is 62\%.

The solid line in Fig. 1 corresponds to the best-fit values of the parameters in Eq. (9), while the shadowed area denotes $3 \sigma$ C.L. region of the fit. One can see that the linear fit has small uncertainties, especially for $Q^{2} \lesssim 3 \mathrm{GeV}^{2}$, where there are many data points.

The best-fit values of the parameters $c_{0}$ and $c_{1}$ in Eq. (9) are close to those obtained by Arrington in Ref. [16], $c_{0}^{\text {bf }}=1.0324$ and $c_{1}^{\mathrm{bf}}=-0.135$. Notice that in Ref. [16] it was assumed that for $Q^{2}<0.24 \mathrm{GeV}^{2}$ the form factor ratio is equal to unity.

\section{B. Fit of polarization and cross section data}

The values of the proton form factors have been extracted from the data of many elastic $e p$ scattering experiments using the Rosenbluth method. In the one-photon approximation, the differential cross section in the laboratory frame for unpolarized $e p$ elastic scattering reads (in the same notation used in the previous subsection)

$$
\begin{aligned}
\sigma\left(E, Q^{2}\right) & \equiv \frac{d \sigma_{1 \gamma}}{d \cos \theta} \\
& =\sigma_{\mathrm{M}}\left(E, Q^{2}\right)\left(G_{E p}^{2}+\frac{\tau}{\epsilon} G_{M p}^{2}\right)\left(\frac{1}{1+\tau}\right),
\end{aligned}
$$

\footnotetext{
${ }^{1}$ Detailed numbers of the covariance matrices relative to these and to the following fit parameters can be found in Ref. [37].
}

$\sigma_{\mathrm{M}}$ being the Mott's differential cross section

$$
\sigma_{\mathrm{M}}\left(E, Q^{2}\right) \equiv\left(\frac{d \sigma}{d \cos \theta}\right)_{\mathrm{M}}=\frac{\pi \alpha^{2} E^{\prime} \cos ^{2}(\theta / 2)}{2 E^{3} \sin ^{4}(\theta / 2)} .
$$

The Rosenbluth separation is then obtained by considering the reduced differential cross section

$$
\begin{aligned}
\sigma_{\mathrm{R}}\left(E, Q^{2}\right) & \equiv \epsilon(1+\tau) \frac{\sigma\left(E, Q^{2}\right)}{\sigma_{\mathrm{M}}\left(E, Q^{2}\right)} \\
& =\tau G_{M p}^{2}\left(Q^{2}\right)+\epsilon G_{E p}^{2}\left(Q^{2}\right) .
\end{aligned}
$$

A linear fit of the reduced differential cross section at fixed $Q^{2}$ and different values of $\epsilon$ gives the value of $\tau G_{M p}^{2}\left(Q^{2}\right)$ from the intercept $(\epsilon=0)$ and the value of $G_{E p}^{2}\left(Q^{2}\right)$ from the slope. Notice, however, that the measurement of $G_{E p}^{2}\left(Q^{2}\right)$ with the Rosenbluth method has large uncertainties, because the contribution of $G_{E p}^{2}\left(Q^{2}\right)$ to the reduced differential cross section in Eq. (13) is suppressed for large values of $Q^{2}(\tau \gtrsim \epsilon)$ while for small values of $Q^{2}$ we have $G_{E p}^{2} \simeq G_{M p}^{2} / \mu_{p} \simeq G_{M p}^{2} / 7.8$.

In our analysis, in the first fit, later called fit I, we assume that $G_{E p}$ is related to $G_{M p}$ by the linear relation of Eq. (7), which is favored by the direct measurement of $\mathcal{R}\left(Q^{2}\right)$ in polarization experiments, as discussed in Sec. II A.

For the proton magnetic form factors, we adopt the parametrization proposed by Kelly [18]:

$$
\frac{G_{M p}\left(Q^{2}\right)}{\mu_{p}}=\frac{1+\sum_{k=1}^{n} a_{p, k}^{M} \tau^{k}}{1+\sum_{k=1}^{n+2} b_{p, k}^{M} \tau^{k}},
$$

which guarantees the asymptotic behavior $G_{M p}\left(Q^{2}\right) \propto Q^{-4}$ at high $Q^{2}$ [39]. We shall employ the parametrization of Eq. (14) with four parameters $(n=1)$ :

$$
\frac{G_{M p}\left(Q^{2}\right)}{\mu_{p}}=\frac{1+a_{p, 1}^{M} \tau}{1+b_{p, 1}^{M} \tau+b_{p, 2}^{M} \tau^{2}+b_{p, 3}^{M} \tau^{3}} .
$$

We will see that this choice turns out to be quite satisfactory for the description of the data. Moreover a relatively small number of parameters allows a better control of the errors.

We have also performed a fit with both the magnetic and electric proton form factors parametrized by the expression (15). This fit will be called fit II in the following. In this case, the electric form factor reads

$$
G_{E p}\left(Q^{2}\right)=\frac{1+a_{p, 1}^{E} \tau}{1+b_{p, 1}^{E} \tau+b_{p, 2}^{E} \tau^{2}+b_{p, 3}^{E} \tau^{3}} .
$$

In our analysis, we consider similar sets of cross section data as the ones employed by Arrington in Ref. [17], namely, the data from Refs. [40-62]. Some of the data were taken from the JLab data base [63]; we include also data from Ref. [64]. Additionally, we considered the latest data of the JLab experiment [10] in which the cross section was measured with the smallest errors.

We fitted the $e p$ cross section data by minimizing the leastsquares function

$$
\chi_{\mathrm{cs}}^{2}=\sum_{i=1}^{M_{\mathrm{cs}}}\left\{\sum_{j=1}^{N_{i}^{\mathrm{cs}}} \frac{\left[n_{i} \sigma_{i, j}^{\exp }-\sigma\left(E_{i, j}, Q_{i, j}^{2}\right)\right]^{2}}{\left(\Delta \sigma_{i, j}^{\exp }\right)^{2}}+\frac{\left(1-n_{i}\right)^{2}}{\left(\Delta n_{i}\right)^{2}}\right\},
$$


where $M_{\mathrm{cs}}=24$ or $M_{\mathrm{cs}}=28$ are the number of data sets, ${ }^{2} N_{i}^{\mathrm{cs}}$ is the number of points in the $i$ th data set, $n_{i}$ and $\Delta n_{i}$ are the corresponding overall normalization and uncertainty, $\sigma_{i, j}^{\exp }$ is the $j$ th differential cross section point in the $i$ th data set, with electron energy $E_{i, j}$ and four-momentum transfer $Q_{i, j}^{2}, \sigma\left(E_{i, j}, Q_{i, j}^{2}\right)$ is the corresponding differential cross section computed with Eq. (11). The uncertainty $\Delta \sigma_{i, j}$ of $\sigma_{i, j}^{\exp }$ includes the statistical and uncorrelated systematic uncertainties added in quadrature.

We perform a simultaneous fit of the polarization and cross section data by minimizing the sum of the least-square functions, Eqs. (8) and (17):

$$
\chi^{2}=\chi_{\text {rat }}^{2}+\chi_{\mathrm{cs}}^{2}
$$

For fit I, the range of $Q^{2}$ taken into account is

$$
0.1 \mathrm{GeV}^{2} \leqslant Q^{2} \leqslant 6 \mathrm{GeV}^{2},
$$

which corresponds to the interval of $Q^{2}$ values where polarization transfer data are available.

For fit II, we extend the range of $Q^{2}$ down to $Q^{2} \simeq 0$ :

$$
0 \leqslant Q^{2} \leqslant 6 \mathrm{GeV}^{2} \text {. }
$$

Notice that a precise knowledge of the form factors in the low $Q^{2}$ region is of special interest for neutrino-nucleon (-nucleus) scattering processes. In both cases, the upper limit, $Q^{2}=6 \mathrm{GeV}^{2}$, is determined by the polarization data. We do not consider higher $Q^{2}$ points.

As already stressed in the literature [11], the inclusion of the most precise data of Ref. [10] definitely indicates the need of corrections to formula (11). Beyond the classical radiative corrections [65], to get agreement with the polarization data, one needs to consider also the two photon exchange (TPE) corrections, which can be written as an additive term to the reduced cross section:

$$
\sigma_{R} \rightarrow \sigma_{R}+\delta_{\mathrm{TPE}}
$$

The calculation of $\delta_{\mathrm{TPE}}$ is difficult and model dependent; however, one can use general properties to derive a phenomenological expression of the TPE term. The scattering amplitude for electron-nucleon interaction must satisfy general symmetry properties, such as crossing symmetry and $C$ invariance [66]. They can be used to constrain $\delta_{\mathrm{TPE}}$.

Following Ref. [67], we adopt a TPE correction given by a function $F\left(Q^{2}, y\right)$, that is,

$$
\sigma_{R} \rightarrow \sigma_{R}+\tau F\left(Q^{2}, y\right)
$$

where

$$
y=\sqrt{\frac{1-\epsilon}{1+\epsilon}} .
$$

The function $F\left(Q^{2}, y\right)$ must satisfy the relation $F\left(Q^{2}, y\right)=$ $-F\left(Q^{2},-y\right)$. The analytical properties of $F\left(Q^{2}, y\right)$ allow one to express this function as a series of odd powers of $y$. Chen et al. [67] truncated the expansion to the second term:

$$
F\left(Q^{2}, y\right)=\alpha G_{D}^{2}\left(Q^{2}\right) y+\beta G_{D}^{2}\left(Q^{2}\right) y^{3},
$$

\footnotetext{
${ }^{2}$ For $Q^{2} \in(0.1,6)$ we have 24 independent data sets, while for $Q^{2} \in(0,6)$ we have 28 independent data sets.
}

$\alpha$ and $\beta$ being fit parameters, and $G_{D}\left(Q^{2}\right)$ the usual dipole form factor

$$
G_{D}\left(Q^{2}\right)=\left(1+\frac{Q^{2}}{M_{V}^{2}}\right)^{-2},
$$

with $M_{V}^{2}=0.71 \mathrm{GeV}^{2}$.

We consider both types of fit; for fit I we obtained

$$
\chi_{\min }^{2} / \mathrm{NDF}=375.97 / 392, \quad \mathrm{GoF}=71 \%,
$$

with the following values for the best-fit parameters:

$$
\begin{gathered}
a_{p, 1}^{M}=1.53 \pm 0.01, \quad b_{p, 1}^{M}=12.87 \pm 0.07, \\
b_{p, 2}^{M}=29.16 \pm 0.25, \quad b_{p, 3}^{M}=41.40 \pm 0.33 \\
c_{0}=1.02 \pm 0.01, \quad c_{1}=-0.13 \pm 0.01 .
\end{gathered}
$$

The parameters of the TPE correction are

$$
\alpha=-0.39 \pm 0.09, \quad \beta=-0.04 \pm 0.09 .
$$

Notice that the values of $c_{0}$ and $c_{1}$ parameters are very similar to those in Eq. (9), obtained by fitting the polarization transfer data alone.

For fit II, the minimization procedure leads to

$$
\chi_{\min }^{2} / \mathrm{NDF}=450.95 / 468, \quad \mathrm{GoF}=71 \%,
$$

with the following values for the fit parameters:

$$
\begin{array}{ll}
b_{p, 1}^{M}=12.31 \pm 0.07, & b_{p, 2}^{M}=25.57 \pm 0.22 \\
b_{p, 3}^{M}=30.61 \pm 0.27, & a_{p, 1}^{M}=1.09 \pm 0.01 \\
b_{p, 1}^{E}=11.12 \pm 0.15, & b_{p, 2}^{E}=15.16 \pm 1.03 \\
b_{p, 3}^{E}=21.25 \pm 3.27, & a_{p, 1}^{E}=-0.19 \pm 0.06 .
\end{array}
$$

The parameters of the TPE correction are

$$
\alpha=-0.36 \pm 0.09, \quad \beta=-0.08 \pm 0.09 \text {. }
$$

We note that from both fits we obtained comparable values of the TPE parameters [see Fig. 2, which illustrates the allowed regions in the $(\alpha, \beta)$ parameter space with a given confidence level (C.L.)]. In both cases, the TPE correction turns out to be negative. Let us mention that the way we introduce the TPE corrections in our analysis also motivates the choice for the upper $Q^{2}$ limit; indeed, following the approach of Ref. [67], the magnitude of TPE is fitted to the data and, in the elastic cross section, it can be comparable to the magnitude of $G_{E p}$. Hence the inclusion of the polarization data (which are less affected by TPE correction) allows a more precise determination of the TPE fit parameters, but restricts the $Q^{2}$ range to the one of the available polarization data.

It is worth mentioning that by excluding the TPE correction [hence using for the cross section formula (11)], both fits worsen, particularly in the goodness of fit. For fit I, we obtain $\chi_{\min }^{2} / \mathrm{NDF}=467.07 / 394$ with $\mathrm{GoF}=0.6 \%$; similarly for fit II we get $\chi_{\min }^{2} / \mathrm{NDF}=544.31 / 470$ with $\mathrm{GoF}=1 \%$. We noticed that this result stems from the presence, in the analysis, of the very accurate JLab data [10], without which GoF would increase to $45 \%$ and $47 \%$, respectively.

In addition to the above discussed form factors, we also checked a different parametrization, based on a two-poles 

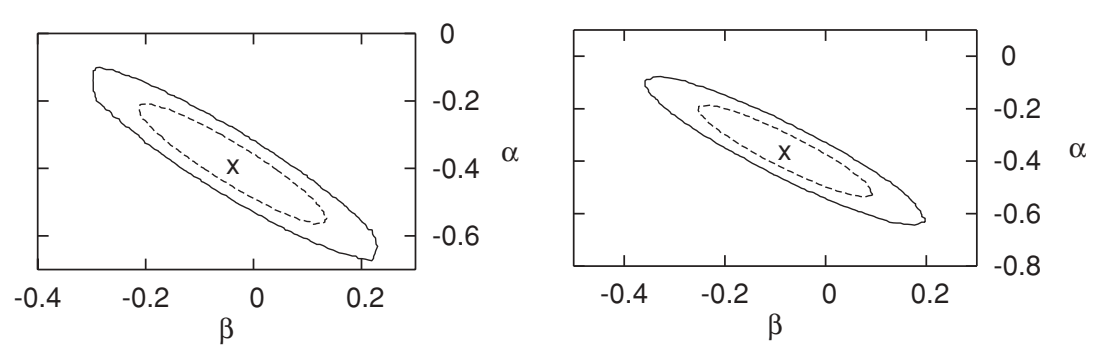

FIG. 2. Projection on the $\alpha-\beta$ parameter space of the contours delimiting the allowed regions in the two-dimensional space of TPE correction parameters, with $1 \sigma$ (dashed lines) and $2 \sigma$ (solid lines) C.L. The contours are computed for fit I (left) and fit II (right). The crosses indicate the projections of the best-fit point, Eq. (28) for fit I and Eq. (31) for fit II. formula for both the electric and magnetic proton form factors [5]:

$$
\begin{aligned}
G_{E p}\left(Q^{2}\right) & =\frac{a_{1}^{E}}{1+a_{2}^{E} Q^{2}}+\frac{1-a_{1}^{E}}{1+a_{3}^{E} Q^{2}}, \\
\frac{G_{M p}\left(Q^{2}\right)}{\mu_{p}} & =\frac{a_{1}^{M}}{1+a_{2}^{M} Q^{2}}+\frac{1-a_{1}^{M}}{1+a_{3}^{M} Q^{2}} .
\end{aligned}
$$

With respect to Kelly's parametrization this would offer the advantage of having a smaller number of parameters, in addition to the ones of the TPE correction. A new, global fit can be obtained with $\chi_{\min }^{2} / \mathrm{NDF}=1.10$, but $\mathrm{GoF}=0.06 \%$, thus indicating that the former parametrization is preferable.

As already mentioned in the Introduction, one of our main tasks is to compute the form factor uncertainties as they can be extracted from the fit. This goal can be achieved by performing an accurate error analysis on the various fit parameters.

We calculated the correlated uncertainties of the fit parameters and the related uncertainties of the form factors with the standard least-squares method, which is appropriate and widely used for nonlinear models ${ }^{3}$ (see Refs. [38,68,69]): the allowed region in the space of $N$ parameters with $\lambda$ confidence level (C.L.) is delimited by the contour defined by

$$
\chi^{2}=\chi_{\min }^{2}+\Delta \chi^{2}(N, \lambda)
$$

\footnotetext{
${ }^{3}$ In this case, the uncertainties of the parameters and their correlations estimated from the covariance matrix are quite approximate. This method was widely used in the past, when computer power was insufficient to perform more accurate evaluations, as the one presented in this paper.
}

where $\Delta \chi^{2}(N, \lambda)$ is the value for which a $\chi^{2}$ variable with $N$ degrees of freedom has a cumulative probability $\lambda$. We consider $2 \sigma(95.45 \%$ C.L.) and $3 \sigma$ (99.73\% C.L.) uncertainties.

Since we have eight parameters in fit I and ten in fit II, the exploration of the parameter space to find the contours defined by Eq. (34) cannot be done with the simplest grid method. Therefore, we used a Monte Carlo Markov chain generator of random points, which allows us to find the allowed parameter regions with good accuracy in a few hours of CPU time of a normal PC.

It is interesting to notice that the estimated values of the magnetic form factor parameters $a_{p, 1}^{M}, b_{p, 1}^{M}, b_{p, 2}^{M}$ and $b_{p, 3}^{M}$ are strongly correlated. In particular, the estimates of $a_{1}$ and $b_{3}$ are almost linearly dependent. These parameters determine the asymptotic behavior of $G_{M p}\left(Q^{2}\right)$, which turns out to be

$$
\lim _{Q^{2} \rightarrow \infty} Q^{4} G_{M p}\left(Q^{2}\right)=\left(4 M^{2}\right)^{2} \frac{a_{p, 1}^{M}}{b_{p, 3}^{M}} \simeq\left(0.68_{-0.01}^{+0.01} \mathrm{GeV}^{2}\right)^{2},
$$

in fair agreement with the one given by the usual dipole form factor (25) (the above uncertainties are at $3 \sigma$ ).

In Fig. 2 (left panel) the error contours for $\alpha$ and $\beta$ parameters are shown. We see that solutions with $\beta$ positive but very small are possible, but in this case $\alpha$ should be negative and large in magnitude. Therefore the TPE corrections are always negative.

In Fig. 3 we show our best fits (fit I) for the magnetic and electric proton form factors with their uncertainties. We compare with Kelly's fit [18]. One can see that Kelly's fit
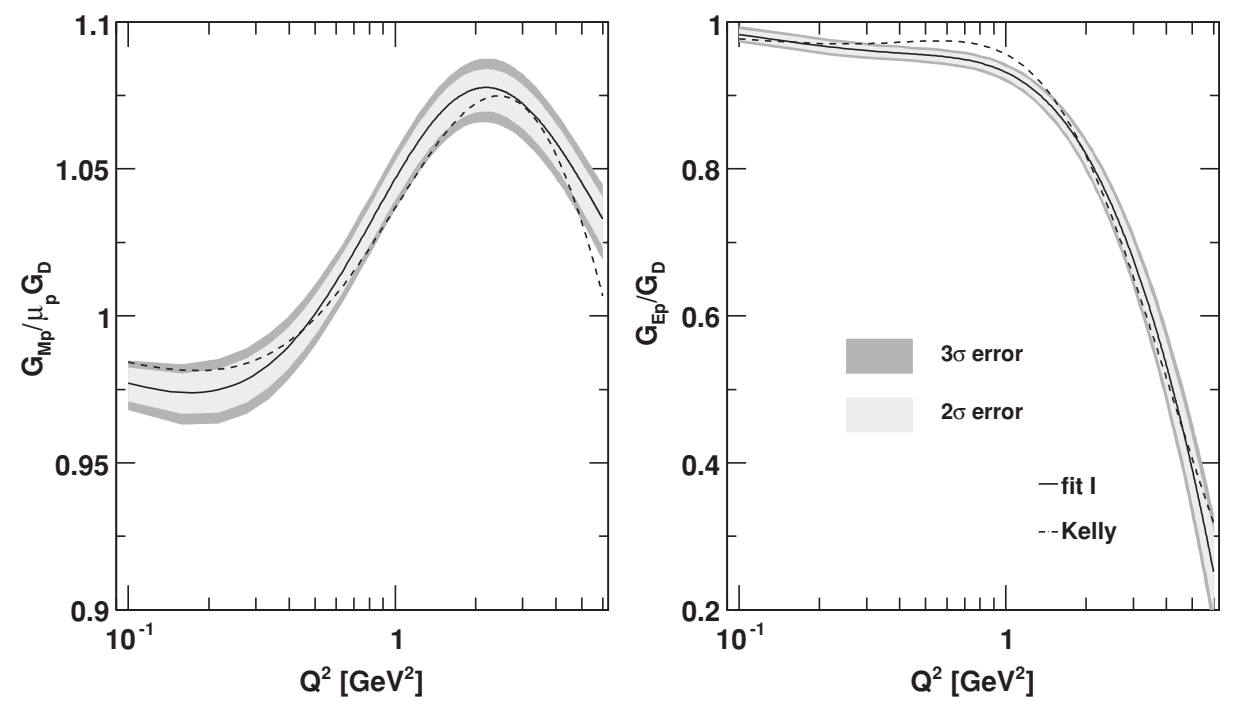

FIG. 3. The fits of $G_{M p} / \mu_{p} G_{D}$ and $G_{E p} / G_{D}$ form factors obtained with fit I and showing the $2 \sigma$ and $3 \sigma$ C.L. allowed regions. Kelly's fit [18] is also shown. 

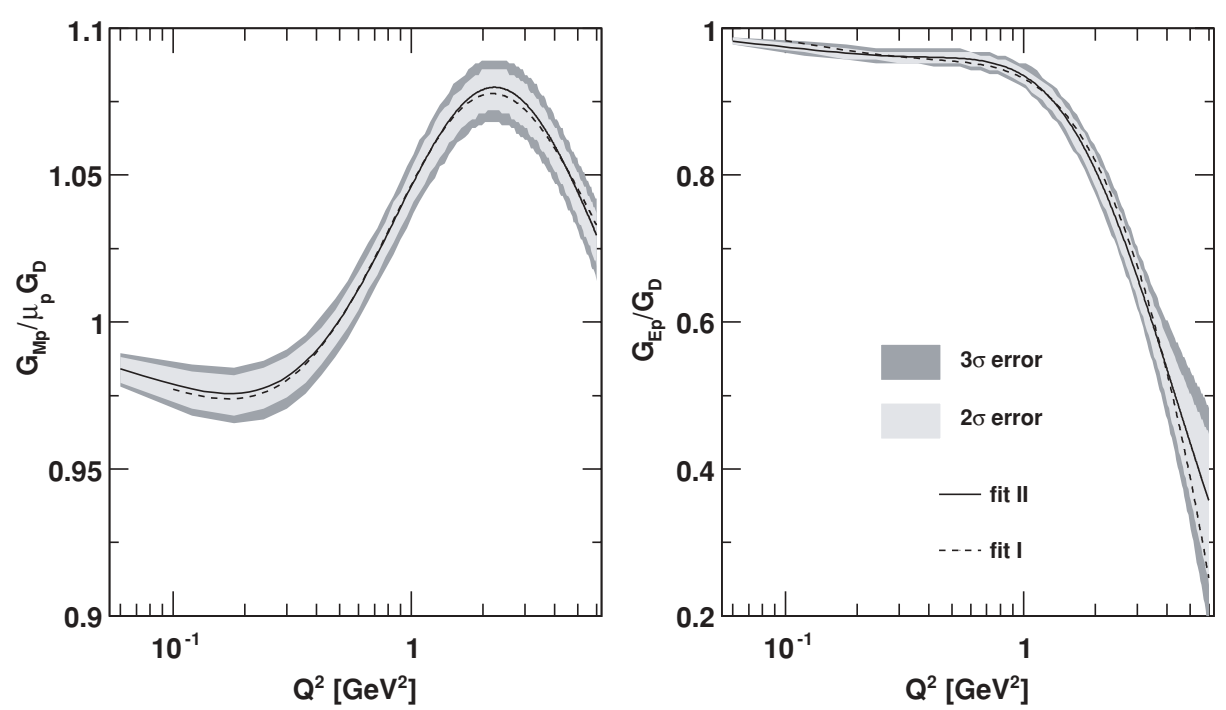

FIG. 4. Electric and magnetic proton form factors obtained with fit II and showing the $2 \sigma$ and $3 \sigma$ C.L. allowed regions. Our previous fit $\mathrm{I}$ is also shown. of the magnetic proton form factor lies within our $3 \sigma$ C.L. region in almost the whole $Q^{2}$ range under consideration. For the electric form factor, the fits differ by more than $3 \sigma$ in a relatively wide range of $Q^{2}$.

A similar error analysis is performed for fit II. Here, the number of form factor parameters is larger (four parameters for each form factor). As above, we show a contour plot for the TPE correction parameters (Fig. 2, right panel).

Analogous to the case of fit I, the estimates of the parameters $a_{p, 1}^{M}$ and $b_{p, 3}^{M}$ are linearly dependent, and their ratio is similar:

$$
\lim _{Q^{2} \rightarrow \infty} Q^{4} G_{M p}\left(Q^{2}\right)=\left(4 M^{2}\right)^{2} \frac{a_{p, 1}^{M}}{b_{p, 3}^{M}} \simeq\left(0.66_{-0.02}^{+0.02} \mathrm{GeV}^{2}\right)^{2}
$$

One could also derive from the form factor parameters the charge and magnetic rms radii for the proton, as given by the slope of the electric and magnetic form factors at $Q^{2}=0$. They turn out to be $\sqrt{\left\langle r_{E p}^{2}\right\rangle}=0.87 \pm 0.01 \mathrm{fm}$ and $\sqrt{\left\langle r_{M p}^{2}\right\rangle}=$ $0.86 \pm 0.01 \mathrm{fm}$. These results are comparable with previous analyses in the literature, but slightly lower than the most recent and advanced estimates of Refs. [70,71]; indeed, the latter take into account Coulomb distortion, which is relevant at low $Q^{2}$. For example, Ref. [71] provides $\sqrt{\left\langle r_{E p}^{2}\right\rangle}=0.895 \pm 0.018 \mathrm{fm}$. The present fit of the form factors is carried out in a planewave approximation, and low- $Q^{2}$ properties like charge and magnetic radii are not properly reproduced without accounting for radiative corrections to the Rosenbluth cross sections.

In Fig. 4 we present $G_{M p}$ and $G_{E p}$ obtained in fit II, with the $2 \sigma$ and $3 \sigma$ C.L. error bands. Here the results from fit I are also plotted. Both fits lead to very similar magnetic form factors. On the contrary, there is a visible difference between the corresponding electric form factors: the $G_{E p}$ obtained in fit I decreases faster then the one obtained in fit II.

Figure 5 shows the ratio $\mu_{p} G_{E p} / G_{M p}$ obtained with fits I and II. The linear ratio fitted only to the polarization data is no longer shown, since it is very similar to the one obtained with fit I. The ratio uncertainties are larger for fit II than for fit I, because the parametrization in fit II contains a larger number of degrees of freedom.
Finally, taken for granted that they are necessary, it is interesting to understand what is the quantitative impact of the TPE corrections: they are expected to be relevant especially for the electric form factors. For this purpose, we compare in Fig. 6 the proton form factors obtained with and without TPE correction (only fit II is considered). The magnetic proton form factor obtained without TPE is systematically shifted down by about $1.5 \%$, and above $Q^{2} \simeq 1 \mathrm{GeV}^{2}$ it lies outside the $3 \sigma$ C.L. region of the form factor obtained by including the TPE correction. The analogous effect on the electric form factor is shown in the middle panel of the same figure and appears to be less uniform than for the magnetic form factor; this can be better appreciated from the bottom panel of Fig. 6, where the ratio

$$
G_{E p}\left(\text { without TPE) } / G_{E p}(\text { with TPE) }\right.
$$

is plotted. One can see that the TPE correction substantially alters the $Q^{2}$ dependence of the electric form factor, in

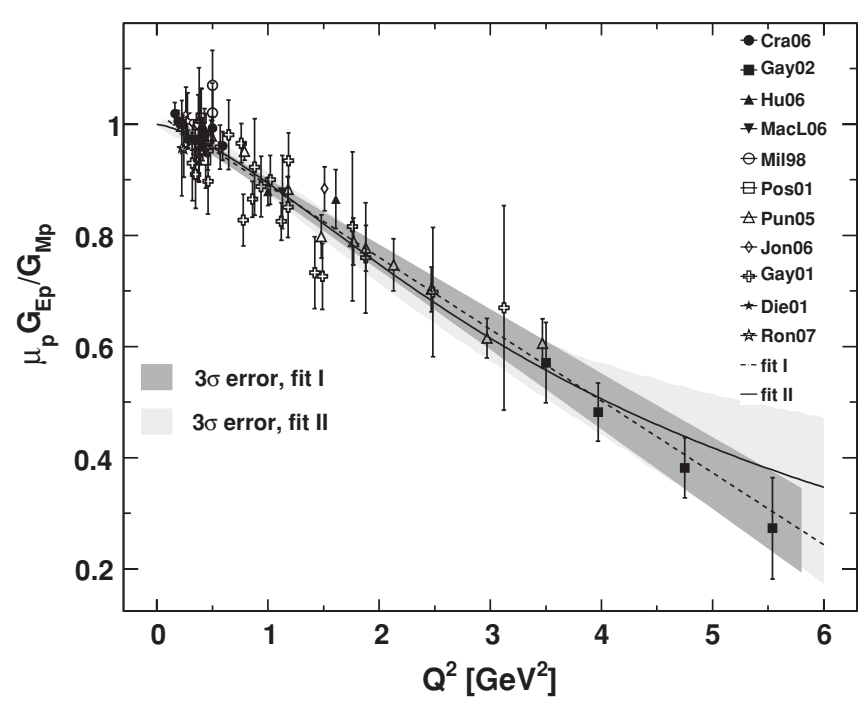

FIG. 5. Ratio $\mu_{p} G_{E p} / G_{M p}$ obtained in the simultaneous fit to polarization measurements and cross section data. Fits I and II are shown with their corresponding $3 \sigma$ C.L. allowed regions. 

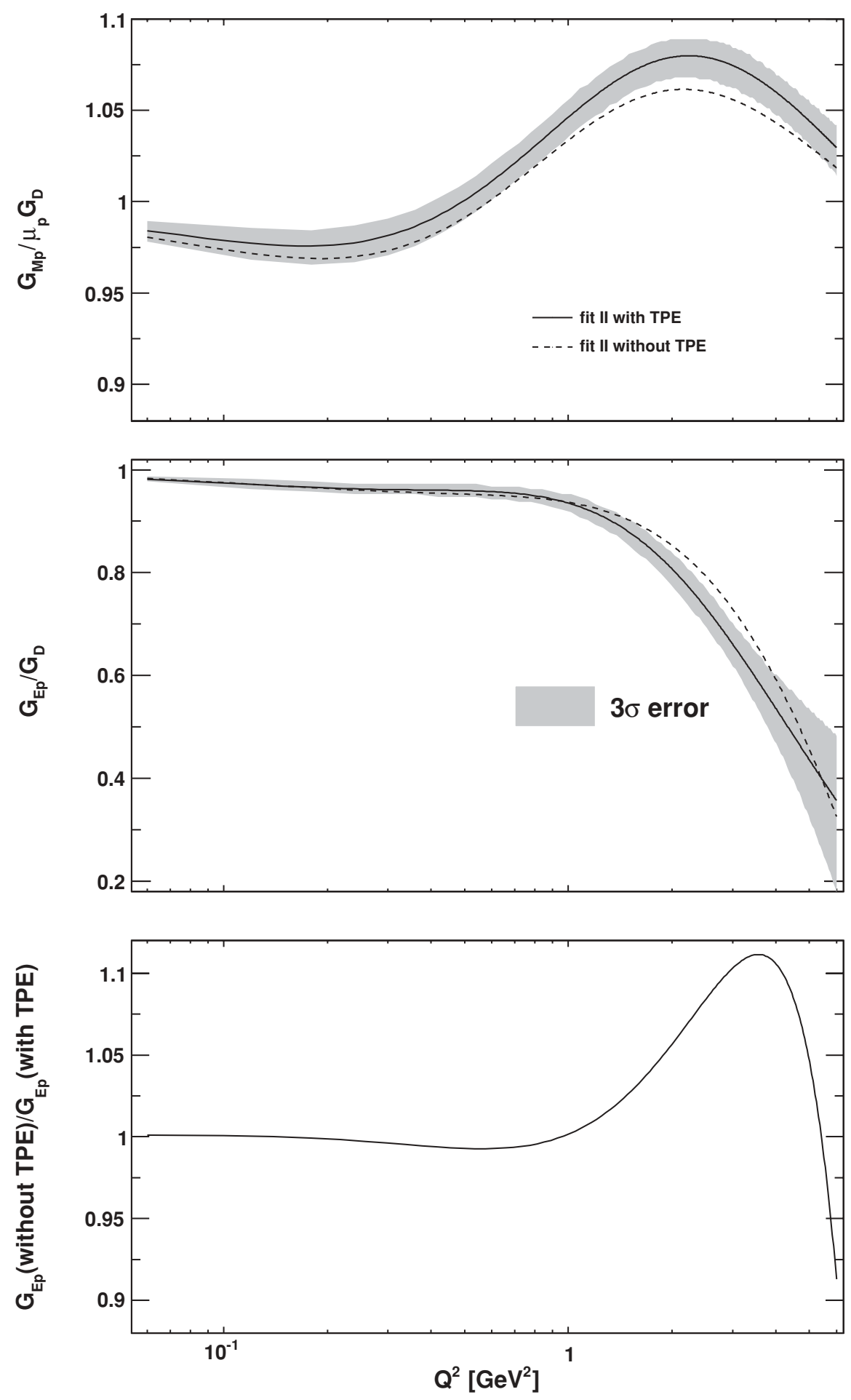

FIG. 6. Comparison of fits obtained with and without TPE correction (solid and dashed lines correspondingly). In the first and second panels, $G_{M p} /\left(\mu_{p} G_{D}\right)$ and $G_{E p} / G_{D}$ are plotted, respectively. The shadowed areas denote the $3 \sigma$ allowed regions. In the bottom panel, the ratio (37) is shown. Results are obtained for fit II. particular, for $Q^{2}>2 \mathrm{GeV}^{2}$, with an effect which grows up to the order of $10 \%$. In any case, the impact of the TPE correction turns out to be non-negligible for both form factors.

\section{NEUTRON FORM FACTORS}

The measurement of the neutron form factors is much more difficult than that of the proton form factors, since a target of free neutrons does not exist. The neutron form factors are extracted from measurements of electron-nucleus scattering, usually electron-deuteron or electron-helium scattering. Therefore, the data analysis is affected by uncertainties stemming from the nuclear theoretical model assumed to describe the target nucleus. Since these models have consistently improved with time, in our analysis we consider only relatively recent data. At variance with the proton case, we take directly from the literature the published values of neutron form factors "data" and apply our fitting procedure to them. 


\section{A. Electric neutron form factor}

For the electric neutron form factor, we adopt the Galsterlike parametrization

$$
G_{E n}\left(Q^{2}\right)=\frac{A \tau}{1+B \tau} G_{D}\left(Q^{2}\right),
$$

with the dipole form factor of Eq. (25).

We consider the electric neutron form factors "data" which have been published in several papers. Some of the data have been obtained in asymmetry and recoil polarization measurements [72-85]. We consider also the reanalyzed electrondeuteron data [86] and the newest BLAST measurements [87]. Additionally, to have a proper slope of the electric form factor in the limit $Q^{2} \rightarrow 0$, we impose to our fit the additional constrain [88]

$$
\left\langle r_{E n}^{2}\right\rangle=-0.1148 \pm 0.0035 \mathrm{fm}^{2} .
$$

We considered a least-squares function similar to the one in Eq. (8), with the experimental statistical and systematical uncertainties added in quadrature. With the values

$$
A=1.68 \pm 0.05, \quad B=3.63 \pm 0.39,
$$

we obtained

$$
\chi_{\min }^{2} / \mathrm{NDF}=25.82 / 37,
$$

and the goodness of the fit turned out to be excellent: $91 \%$.

In Fig. 7 we plot the best-fit value of $G_{E n}$ as a function of $Q^{2}$ together with the $2 \sigma$ and $3 \sigma$ C.L. allowed regions. We plot also Kelly's fit [18].

As an alternative to the most commonly used Galster-like parametrization, we considered a neutron electric form factor given by the sum of two dipole form factors:

$$
G_{E n}\left(Q^{2}\right)=\frac{a}{\left(1+b_{1} Q^{2}\right)^{2}}-\frac{a}{\left(1+b_{2} Q^{2}\right)^{2}} .
$$

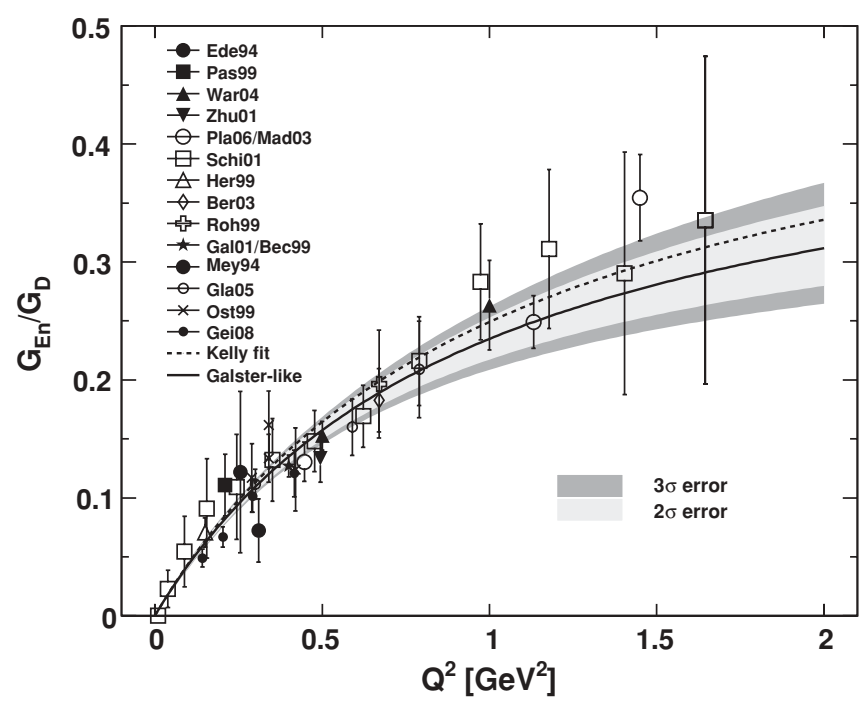

FIG. 7. Fit of the electric neutron form form factor (solid line) obtained with the Galster-like parametrization [Eq. (38)]. The shadowed areas denote the $2 \sigma$ and $3 \sigma$ C.L. allowed regions. Kelly's fit [18] is also shown.

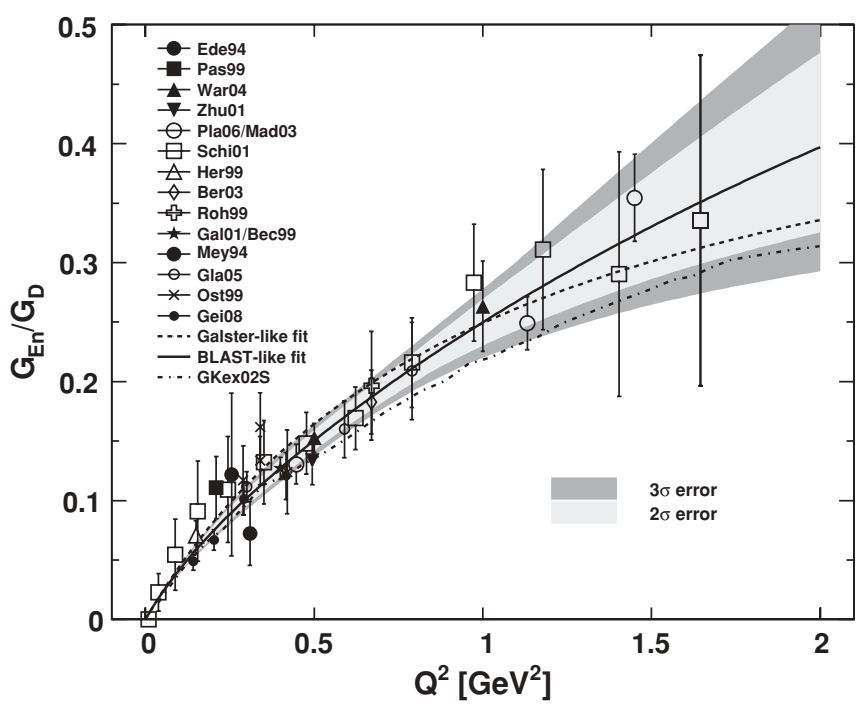

FIG. 8. Fit of the electric neutron form form factor (solid line). The BLAST-like parametrization (42) is shown with the shadowed $2 \sigma$ and $3 \sigma$ C.L. allowed regions. Also shown are the Galster-like parametrization and the Lomon result (GKex02S) [22].

This parametrization is similar to the one considered in the latest BLAST data analysis [87], and for this reason we will call it the BLAST-like parametrization. The fitting procedure for the above parametrization leads to

$$
\chi^{2} / \mathrm{NDF}=17.95 / 36, \quad \mathrm{GoF}=99 \%,
$$

with the parameters

$a=-0.10 \pm 0.02, \quad b_{1}=2.83 \pm 0.37, \quad b_{2}=0.43 \pm 0.11$.

In Fig. 8 the BLAST-like parametrization is compared with the data from the Galster-like parametrization and a recent Lomon parametrization $[21,22]$ (GKex02S). Notice that the parametrization (44) rises faster with $Q^{2}$ than the Galster-like or the Lomon one, but the latter remain both within the BLAST parametrization uncertainties.

\section{B. Magnetic neutron form factor}

For the neutron magnetic form factor, we adopted again the simplest form of Kelly's parametrizations, with $n=1$,

$$
\frac{G_{M n}\left(Q^{2}\right)}{\mu_{n}}=\frac{1+a_{n, 1}^{M} \tau}{1+b_{n, 1}^{M} \tau+b_{n, 2}^{M} \tau^{2}+b_{n, 3}^{M} \tau^{3}} .
$$

We considered 11 data sets, obtained from asymmetry measurements [89-93] and cross section measurements in electron-deuterium scattering [94-103], where Ref. [103] contains the latest JLab measurements. The fit to all these data sets leads, however, to a minimum $\chi^{2} / \mathrm{NDF}=2.05$, not quite satisfactory. According to Kelly [70], the data from Refs. [101] and [97] were extracted using the same associated-particle 


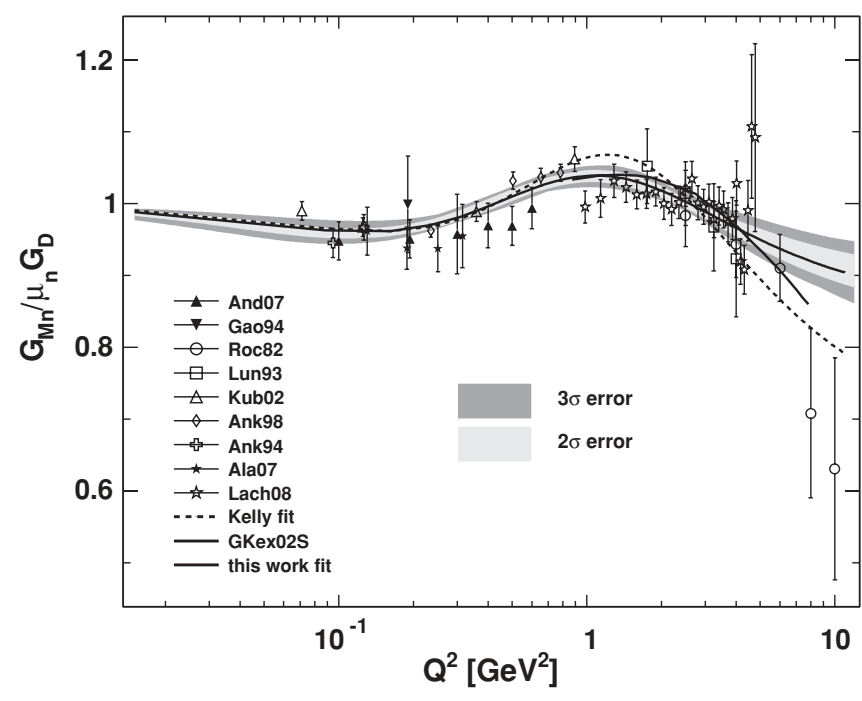

FIG. 9. Fit of magnetic neutron form factor (solid line), normalized to the dipole form factor, and showing the $2 \sigma$ and $3 \sigma$ C.L. allowed regions. Kelly's fit [18] is also shown.

technique for the neutron efficiency, a technique that appears to be in contradiction with the method used in other experiments. Therefore we omitted those two data sets from our final analysis. After excluding the two above mentioned data sets, we obtained a fit over $N=56$ points with

$$
\chi_{\min }^{2} / \mathrm{NDF}=52.79 / 52=1.01,
$$

and $\mathrm{GoF}=44 \%$. The corresponding values for the parameters in the neutron magnetic form factor are

$$
\begin{aligned}
& b_{n, 1}^{M}=21.30 \pm 4.56, \quad b_{n, 2}^{M}=77 \pm 31, \\
& b_{n, 3}^{M}=238 \pm 105, \quad a_{n, 1}^{M}=8.28 \pm 3.89 .
\end{aligned}
$$

We performed the error analysis over the four parameters above. Even if the fit of $G_{M n}$ was done on a slightly different basis than the one of the proton, we still observe strong correlations between estimated values of the parameters, in full analogy with our findings for the proton form factor. In particular, the estimated values of parameters $\left(a_{1}\right.$ and $\left.b_{3}\right)$ which determine the asymptotic behavior of the form factor at large $Q^{2}$ are almost linearly dependent:

$$
\lim _{Q^{2} \rightarrow \infty} Q^{4} G_{M n}\left(Q^{2}\right)=\left(4 M^{2}\right)^{2} \frac{a_{n, 1}^{M}}{b_{n, 3}^{M}} \simeq\left(0.66_{-0.01}^{+0.01} \mathrm{GeV}^{2}\right)^{2} .
$$

This value is very similar to the one obtained for the proton. Notice, however, that without the newest JLab data, instead of the value in Eq. (48) we would get $\left(0.58_{-0.05}^{+0.04} \mathrm{GeV}^{2}\right)^{2}$. In Fig. 9, our final fit of $G_{M n}$ is different from Kelly's result [18], since it contains the newest JLab measurements.

\section{DISCUSSION AND CONCLUSIONS}

In this section, we start by presenting further comparisons of the form factors resulting from our fits with the ones of previous data analyses. In Ref. [11] the first systematic global analyses of the cross section and polarization transfer data on the proton with the inclusion of the TPE correction was performed. That fit is valid up to $Q^{2}=30 \mathrm{GeV}^{2}$ for the magnetic form factor and up to $6 \mathrm{GeV}^{2}$ for the electric form factor. In Fig. 10 we display together our global fits and those of Ref. [11]: it clearly appears that, even though different approaches for the TPE correction were employed, the global fits are very similar.

We also compare our fits with the recent one of Bodek et al. [20] (BBBA07). This global fit is tailored to accurately describe the form factors at low $Q^{2}$ as well as in the intermediate region of $Q^{2}$. These authors used Kelly's parametrization with four parameters, but each form factor was multiplied by some Legendre polynomial, which depends on several additional parameters, constrained to reproduce the low $Q^{2}$ behavior obtained in Ref. [19].

The authors of Ref. [20] plotted the form factors against the so-called Nachtman variable, which for elastic scattering is defined as $\xi=2 /(1+\sqrt{1+1 / \tau})$. Therefore to make the comparison with their results, we express our form factors in
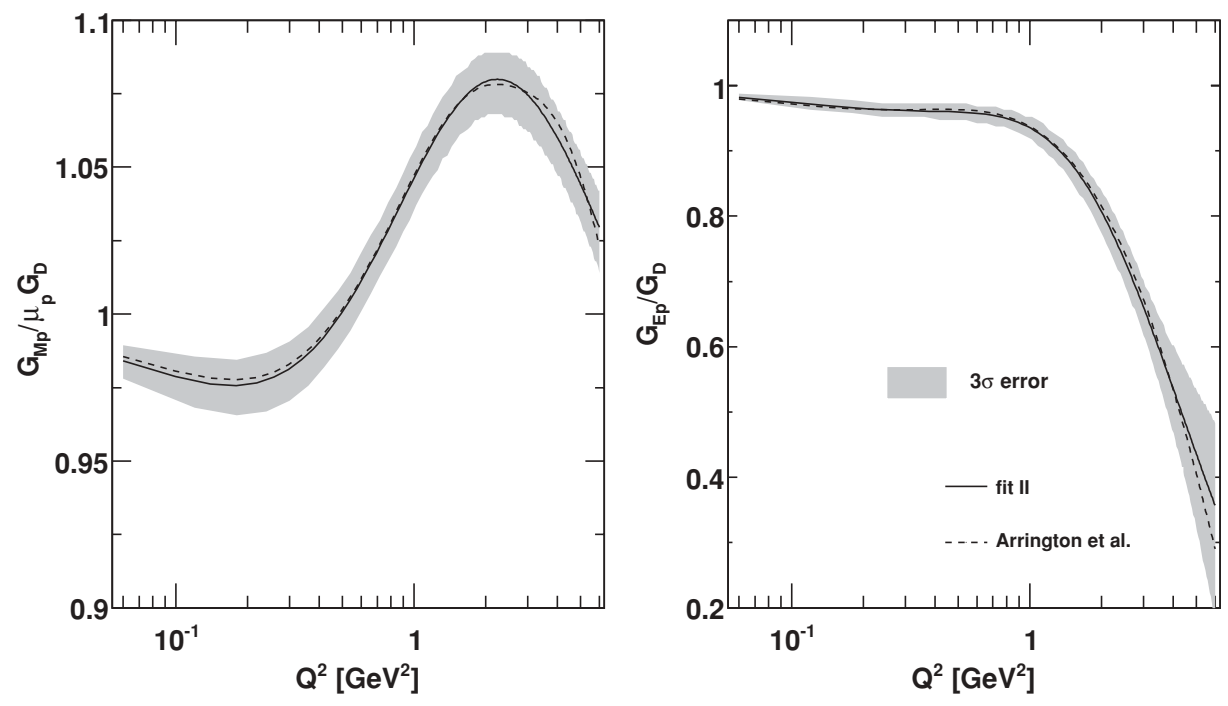

FIG. 10. Magnetic and electric proton form factors (normalized to the dipole form factor). The comparison between fit I (solid lines) and Arrington et al. [11] (dashed lines) is shown. The shadowed areas denote the $3 \sigma$ C.L. allowed region. 

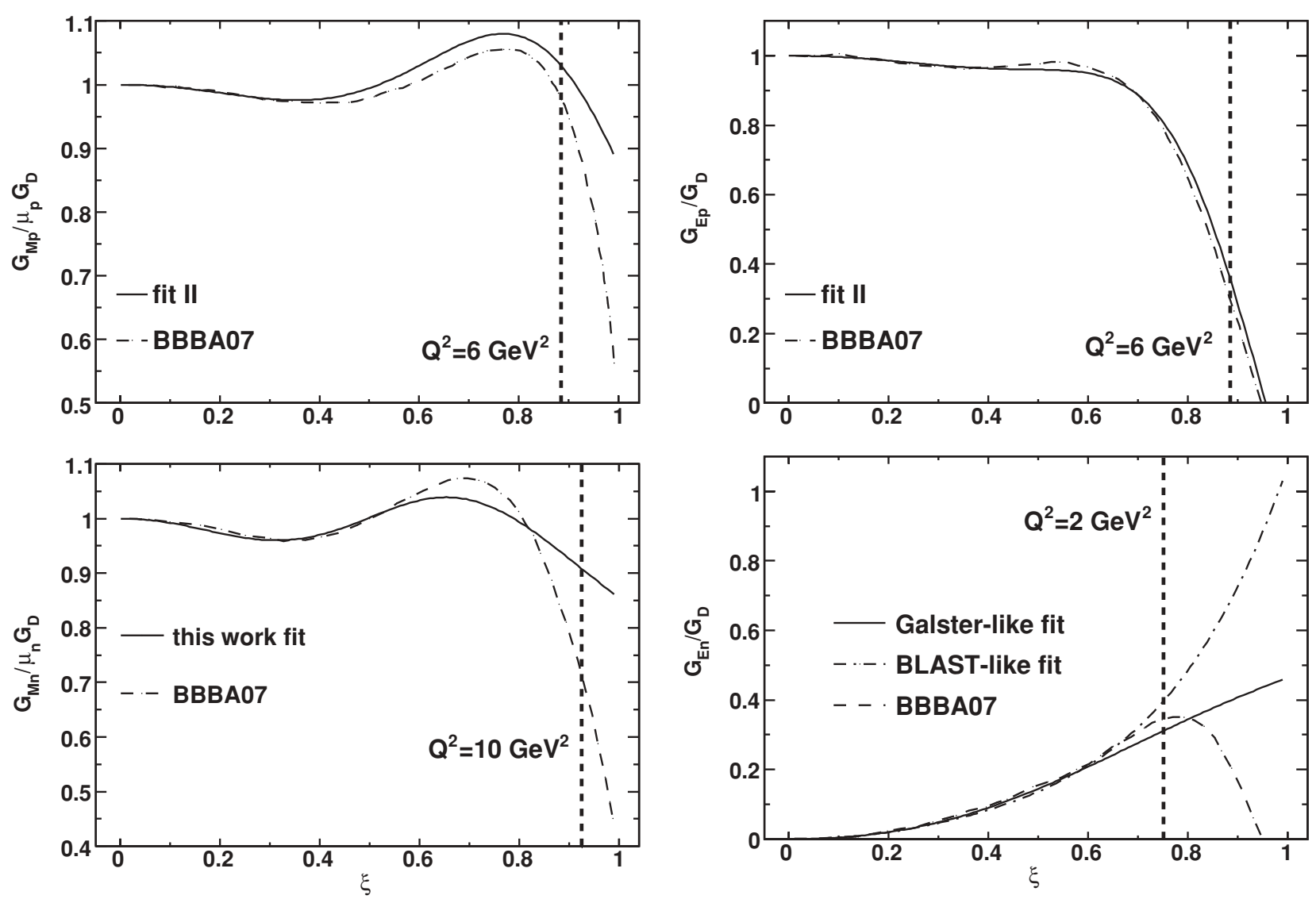

FIG. 11. Proton and neutron electric and magnetic form factors (normalized to the dipole form factor). The fits of electric and magnetic form factors (fit II) as well as the fit of the neutron magnetic form factor are denoted by solid lines. BBBA07 [20] global fits are denoted by dashed lines. In the case of the electric neutron form factor, both parametrizations, Galster and BLAST-like, are shown by the solid and dash-dotted lines, respectively. The form factors are plotted against the Nachtman variable. The dashed vertical bar represents the upper bound of the $Q^{2}$ range where our fits were performed.

terms of the $\xi$ variable. Plots are shown in Fig. 11. In the region of $\xi$ corresponding to the range of validity of our fits, the predictions of the two parametrizations are very similar; however, our magnetic proton form factor is systematically higher (by several percent) then the one given by the BBBA07 parametrization. The difference is given by the TPE correction that we considered in our fitting procedure (see Fig. 6), while authors of Ref. [20] did not discuss this effect. For higher $\xi$ values, one can notice sizable deviations for our form factors. However, we notice, that the $\xi$ variable compresses in a very short range the large $Q^{2}$ region.

Finally we compare our estimates of uncertainties with those obtained by Arrington and Sick [19], who made a serious attempt to compute the uncertainties of the nucleon form factors, which is crucial information in the study of parity-violating $e p$ scattering. Their method to compute errors is explained in Ref. [19] and differs from ours, particularly in the treatment of the systematic uncertainties. In particular, we usually obtain asymmetric uncertainties around the best-fit value. Hence in Fig. 12 we compare the errors of Arrington and Sick on the electric and magnetic proton form factors with our lower and upper bounds for the $3 \sigma$ confidence level errors. For the magnetic proton form factor, within the $3 \sigma$ C.L. our results are consistent with those of Ref. [19]. For the electric proton form factor, we notice some deviations between our results and the ones obtained by Arrington and Sick.

In conclusion, we have presented two fits of the proton and neutron electromagnetic form factors, using the best available data. The $e p$ elastic cross sections were reproduced by including a simple but realistic parametrization of the two photon exchange correction. Alternative parametrizations with fewer parameters than the one employed here do not obtain equally good fits. We show that the impact of the TPE correction on the magnetic and electric proton form factors is larger than the $3 \sigma$ uncertainty of the fits (in a wide range of $Q^{2}$ ). In fit I, we constrained the electric proton form factor by the ratio $\mu_{p} G_{E p} / G_{M p}$ extracted from recoil polarization and asymmetry data. Fit II employs Kelly's parametrization with four parameters, both for the electric and magnetic proton form factors. This fit is obtained with two additional parameters with respect to fit I; however, we believe that it is more reliable than the former, particularly in the low $Q^{2}$ region. We also performed a careful analysis of the uncertainties resulting on 

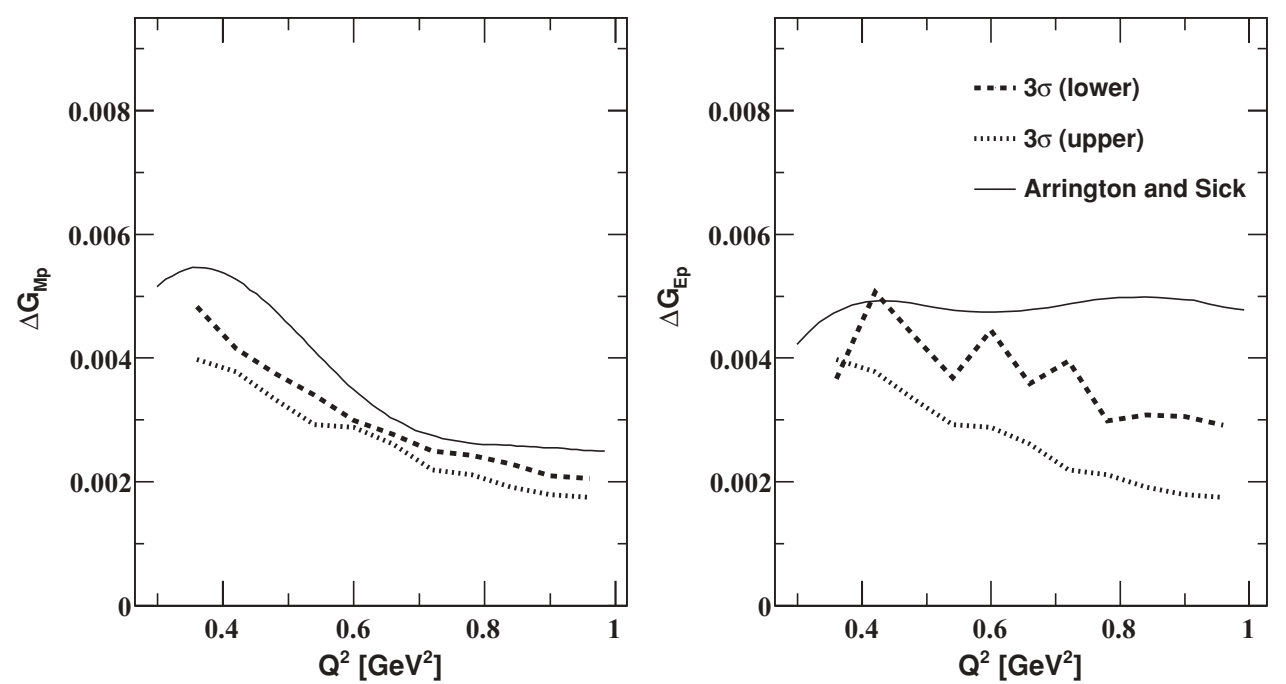

FIG. 12. Uncertainties of electric and magnetic proton form factors. Our computations (fit II) are denoted by dotted (upper uncertainties) and dashed lines (lower uncertainties) for the $3 \sigma$ C.L. errors. The solid lines correspond to the results obtained in Ref. [19]. the parameters of the fit and hence on the form factors. It is worth stressing that only a few papers, among the many devoted to the nucleon electromagnetic form factors, include the TPE correction in the analysis. As a final remark, we remind the reader that even small uncertainties in the magnetic form factors of the proton and neutron turn out to be important for a correct analysis of the neutrino-nucleon cross sections.

The numerical results of our fits are available on the web site [37].

\section{ACKNOWLEDGMENTS}

The authors acknowledge fruitful discussions with E. L. Lomon and T. W. Donnelly. K.M.G. thanks Ron Gilman for his remarks on the previous version of the manuscript, which helped to improve the present one. S.M.B. was supported by the Munich Cluster of Excellence (Origin and Structure of the Universe), the DFG (Transregio 27: Neutrinos and Beyond), and the INFN Sezione di Torino. K.M.G. was supported by the WWS project.
[1] H. y. Gao, Int. J. Mod. Phys. E 12, 1 (2003); 12, 567(E) (2003).

[2] J. Arrington, C. D. Roberts, and J. M. Zanotti, J. Phys. G 34, S23 (2007).

[3] C. F. Perdrisat, V. Punjabi, and M. Vanderhaeghen, Prog. Part. Nucl. Phys. 59, 694 (2007).

[4] R. Gran et al. (K2K Collaboration), Phys. Rev. D 74, 052002 (2006).

[5] W. M. Alberico, S. M. Bilenky, C. Giunti, and C. Maieron, Z. Phys. C 70, 463 (1996).

[6] P. A. M. Guichon and M. Vanderhaeghen, Phys. Rev. Lett. 91, 142303 (2003)

[7] P. G. Blunden, W. Melnitchouk, and J. A. Tjon, Phys. Rev. Lett. 91, 142304 (2003).

[8] Y. C. Chen, A. Afanasev, S. J. Brodsky, C. E. Carlson, and M. Vanderhaeghen, Phys. Rev. Lett. 93, 122301 (2004).

[9] A. V. Afanasev, S. J. Brodsky, C. E. Carlson, Y. C. Chen, and M. Vanderhaeghen, Phys. Rev. D 72, 013008 (2005).

[10] I. A. Qattan et al., Phys. Rev. Lett. 94, 142301 (2005).

[11] J. Arrington, W. Melnitchouk, and J. A. Tjon, Phys. Rev. C 76, 035205 (2007).

[12] C. E. Carlson and M. Vanderhaeghen, Annu. Rev. Nucl. Part. Sci. 57, 171 (2007).

[13] P. E. Bosted, Phys. Rev. C 51, 409 (1995).

[14] E. J. Brash, A. Kozlov, S. Li, and G. M. Huber, Phys. Rev. C 65, 051001(R) (2002).

[15] H. Budd, A. Bodek, and J. Arrington, presented at 2nd International Workshop on Neutrino-Nucleus Interactions in the Few GeV Region (NUINT 02), Irvine, CA, 12-15 December 2002, arXiv:hep-ex/0308005.
[16] J. Arrington, Phys. Rev. C 68, 034325 (2003).

[17] J. Arrington, Phys. Rev. C 69, 032201(R) (2004).

[18] J. J. Kelly, Phys. Rev. C 70, 068202 (2004).

[19] J. Arrington and I. Sick, Phys. Rev. C 76, 035201 (2007).

[20] A. Bodek, S. Avvakumov, R. Bradford, and H. Budd, Eur. Phys. J. C 53, 349 (2008).

[21] E. L. Lomon, Phys. Rev. C 64, 035204 (2001).

[22] E. L. Lomon, arXiv:nucl-th/0609020.

[23] S. Galster, H. Klein, J. Moritz, K. H. Schmidt, D. Wegener, and J. Bleckwenn, Nucl. Phys. B32, 221 (1971).

[24] A. F. Krutov and V. E. Troitsky, Eur. Phys. J. A 16, 285 (2003).

[25] B. D. Milbrath et al. (Bates FPP Collaboration), Phys. Rev. Lett. 80, 452 (1998); 82, 2221(E) (1999).

[26] M. K. Jones et al. (Jefferson Lab Hall A Collaboration), Phys. Rev. Lett. 84, 1398 (2000).

[27] S. Dieterich et al., Phys. Lett. B500, 47 (2001).

[28] T. Pospischil et al. (A1 Collaboration), Eur. Phys. J. A 12, 125 (2001).

[29] O. Gayou et al., Phys. Rev. C 64, 038202 (2001).

[30] O. Gayou et al. (Jefferson Lab Hall A Collaboration), Phys. Rev. Lett. 88, 092301 (2002).

[31] V. Punjabi et al., Phys. Rev. C 71, 055202 (2005); 71, 069902(E) (2005).

[32] C. B. Crawford et al., Phys. Rev. Lett. 98, 052301 (2007).

[33] B. Hu et al., Phys. Rev. C 73, 064004 (2006).

[34] M. K. Jones et al. (Resonance Spin Structure Collaboration), Phys. Rev. C 74, 035201 (2006).

[35] G. MacLachlan et al., Nucl. Phys. A764, 261 (2006).

[36] G. Ron et al., Phys. Rev. Lett. 99, 202002 (2007). 
[37] http://www.nu.to.infn.it/pap/08/ff/ff.php.

[38] C. Amsler et al. (Particle Data Group), Phys. Lett. B667, 1 (2008).

[39] S. J. Brodsky and G. R. Farrar, Phys. Rev. Lett. 31, 1153 (1973).

[40] T. Janssens, R. Hofstadter, E. B. Hughes, and M. R. Yearian, Phys. Rev. 142, 922 (1966).

[41] W. Bartel et al., Phys. Rev. Lett. 17, 608 (1966).

[42] W. Albrecht, H. J. Behrend, F. W. Brasse, W. Flauger, H. Hultschig, and K. G. Steffen, Phys. Rev. Lett. 17, 1192 (1966).

[43] W. Albrecht, H. J. Behrend, H. Dorner, W. Flauger, and H. Hultschig, Phys. Rev. Lett. 18, 1014 (1967).

[44] J. Litt et al., Phys. Lett. B31, 40 (1970).

[45] M. Goitein et al., Phys. Rev. D 1, 2449 (1970).

[46] L. E. Price, J. R. Dunning, M. Goitein, K. Hanson, T. Kirk, and R. Wilson, Phys. Rev. D 4, 45 (1971).

[47] C. Berger, V. Burkert, G. Knop, B. Langenbeck, and K. Rith, Phys. Lett. B35, 87 (1971).

[48] F. Borkowski, P. Peuser, G. G. Simon, V. H. Walther, and R. D. Wendling, Nucl. Phys. A222, 269 (1974).

[49] P. N. Kirk et al., Phys. Rev. D 8, 63 (1973).

[50] W. Bartel et al., Nucl. Phys. B58, 429 (1973).

[51] F. Borkowski, P. Peuser, G. G. Simon, V. H. Walther, and R. D. Wendling, Nucl. Phys. B93, 461 (1975).

[52] J. J. Murphy, Y. M. Shin, and D. M. Skopik, Phys. Rev. C 9, 2125 (1974); 10, 2111(E) (1974).

[53] S. Stein et al., Phys. Rev. D 12, 1884 (1975).

[54] G. G. Simon, C. Schmitt, F. Borkowski, and V. H. Walther, Nucl. Phys. A333, 381 (1980).

[55] G. G. Simon, C. Schmitt, and V. H. Walther, Nucl. Phys. A364, 285 (1981).

[56] S. Rock et al., Phys. Rev. D 46, 24 (1992).

[57] A. F. Sill et al., Phys. Rev. D 48, 29 (1993).

[58] R. C. Walker et al., Phys. Rev. D 49, 5671 (1994).

[59] L. Andivahis et al., Phys. Rev. D 50, 5491 (1994).

[60] D. Dutta et al. (JLab E91013 Collaboration), Phys. Rev. C 68, 064603 (2003).

[61] M. E. Christy et al. (E94110 Collaboration), Phys. Rev. C 70, 015206 (2004).

[62] I. Niculescu, Ph.D. thesis, Hampton University, 1999.

[63] http://www.jlab.org/resdata.

[64] R. G. Arnold et al., Phys. Rev. Lett. 57, 174 (1986).

[65] L. W. Mo and Y. S. Tsai, Rev. Mod. Phys. 41, 205 (1969).

[66] M. P. Rekalo and E. Tomasi-Gustafsson, Eur. Phys. J. A 22, 331 (2004).

[67] Y. C. Chen, C. W. Kao, and S. N. Yang, Phys. Lett. B652, 269 (2007).

[68] D. E. Groom et al. (Review of Particle Physics RPP2000), Eur. Phys. J. C 15, 1 (2000).
[69] W. T. Eadie, D. Drijard, F. E. James, M. Roos, and B. Sadoulet, Statistical Methods in Experimental Physics (North-Holland, Amsterdam, 1971).

[70] J. J. Kelly, Phys. Rev. C 66, 065203 (2002).

[71] I. Sick, Phys. Lett. B576, 62 (2003).

[72] J. Bermuth et al., Phys. Lett. B564, 199 (2003).

[73] J. Golak, G. Ziemer, H. Kamada, H. Witala, and W. Glöckle, Phys. Rev. C 63, 034006 (2001).

[74] J. Becker et al., Eur. Phys. J. A 6, 329 (1999).

[75] M. Meyerhoff et al., Phys. Lett. B327, 201 (1994).

[76] D. Rohe et al., Phys. Rev. Lett. 83, 4257 (1999).

[77] H. Zhu et al. (E93026 Collaboration), Phys. Rev. Lett. 87, 081801 (2001).

[78] T. Eden et al., Phys. Rev. C 50, R1749 (1994).

[79] C. Herberg et al., Eur. Phys. J. A 5, 131 (1999).

[80] R. Madey et al. (E93-038 Collaboration), Phys. Rev. Lett. 91, 122002 (2003).

[81] I. Passchier et al., Phys. Rev. Lett. 82, 4988 (1999).

[82] G. Warren et al. (Jefferson Lab E93-026 Collaboration), Phys. Rev. Lett. 92, 042301 (2004).

[83] D. I. Glazier et al., Eur. Phys. J. A 24, 101 (2005).

[84] M. Ostrick et al., Phys. Rev. Lett. 83, 276 (1999).

[85] B. Plaster et al. (Jefferson Laboratory E93-038 Collaboration), Phys. Rev. C 73, 025205 (2006).

[86] R. Schiavilla and I. Sick, Phys. Rev. C 64, 041002(R) (2001).

[87] E. Geis et al. (BLAST Collaboration), Phys. Rev. Lett. 101, 042501 (2008).

[88] S. Kopecky, P. Riehs, J. A. Harvey, and N. W. Hill, Phys. Rev. Lett. 74, 2427 (1995).

[89] B. Anderson et al. (Jefferson Lab E95-001 Collaboration), Phys. Rev. C 75, 034003 (2007).

[90] W. Xu et al., Phys. Rev. Lett. 85, 2900 (2000).

[91] W. Xu et al. (Jefferson Lab E95-001 Collaboration), Phys. Rev. C 67, 012201 (2003).

[92] H. Gao et al., Phys. Rev. C 50, R546 (1994).

[93] R. Alarcon (BLAST Collaboration), Eur. Phys. J. A 31, 588 (2007).

[94] H. Anklin et al., Phys. Lett. B336, 313 (1994).

[95] H. Anklin et al., Phys. Lett. B428, 248 (1998).

[96] W. K. Brooks and J. D. Lachniet (CLAS Collaboration), Nucl. Phys. A755, 261 (2005).

[97] E. E. W. Bruins et al., Phys. Rev. Lett. 75, 21 (1995).

[98] E. E. W. Bruins et al., Phys. Rev. Lett. 79, 5187 (1997).

[99] G. Kubon et al., Phys. Lett. B524, 26 (2002).

[100] A. Lung et al., Phys. Rev. Lett. 70, 718 (1993).

[101] P. Markowitz et al., Phys. Rev. C 48, R5 (1993).

[102] S. Rock et al., Phys. Rev. Lett. 49, 1139 (1982).

[103] J. Lachniet et al. (CLAS Collaboration), Phys. Rev. Lett. 102, 192001 (2009). 